\title{
A multicenter prospective study of patients
} undergoing open ventral hernia repair with intraperitoneal positioning using the monofilament polyester composite ventral patch:
interim results of the PANACEA study

This article was published in the following Dove Press journal:

Medical Devices: Evidence and Research

12 May 2017

Number of times this article has been viewed

\author{
Frederik Berrevoet ${ }^{1}$ \\ Carl Doerhoff ${ }^{2}$ \\ Filip Muysoms ${ }^{3}$ \\ Steven Hopson ${ }^{4}$ \\ Marco Gallinella Muzi ${ }^{5}$ \\ Simon Nienhuijs ${ }^{6}$ \\ Eric Kullman ${ }^{7}$ \\ Tim Tollens ${ }^{8}$ \\ Mark R Schwartz ${ }^{9}$ \\ Karl LeBlanc ${ }^{10}$ \\ Vic Velanovich"1 \\ Lars Nannestad Jørgensen ${ }^{12}$ \\ 'Department of General and \\ Hepatopancreaticobiliary Surgery, Ghent \\ University Hospital, Ghent, Belgium; \\ ${ }^{2}$ General Surgery, Surgicare of Missouri, \\ Jefferson City, MO, USA; ${ }^{3}$ Department of \\ Surgery, AZ Maria Middelares Ghent, Ghent, \\ Belgium; ${ }^{4}$ Bon Secours Hernia Center, Mary \\ Immaculate Hospital, Newport News, VA, \\ USA; ${ }^{5}$ University Hospital Tor Vergata, Rome, \\ Italy; ${ }^{6}$ Catharina Hospital, Eindhoven, the \\ Netherlands; ${ }^{7}$ Medicinskt Centrum Linköping, \\ Linköping, Sweden; ${ }^{8}$ Imelda Hospital-General \\ Surgery Imelda Hospital, Bonheiden, Belgium; \\ ${ }^{9}$ Monmouth Medical Center, Long Branch, \\ NJ, ${ }^{10}$ Our Lady of Lakes Regional Medical \\ Center, Baton Rouge, LA, "'Tampa General \\ Hospital, University of South Florida, Tampa, \\ $\mathrm{FL}$, USA; ${ }^{12}$ Bispebjerg Hospital, University of \\ Copenhagen, Copenhagen, Denmark
}

Correspondence: Frederik Berrevoet

Department of General and

Hepatopancreaticobiliary Surgery, Ghent

University Hospital, De Pintelaan 185, Ghent

9000 , Belgium

Tel +3293324892

Fax +3293323891

Email frederik.berrevoet@ugent.be
Purpose: This study assessed the recurrence rate and other safety and efficacy parameters following ventral hernia repair with a polyester composite prosthesis (Parietex ${ }^{\mathrm{TM}}$ Composite Ventral Patch [PCO-VP]).

Patients and methods: A single-arm, multicenter prospective study of 126 patients undergoing open ventral hernia repair with the PCO-VP was performed. Patient outcomes were assessed at discharge and at 10 days, 1, 6, 12, and 24 months postoperative.

Results: All patients had hernioplasty for umbilical $(n=110,87.3 \%)$ or epigastric hernia $(n=$ $16,12.7 \%$ ). Mean hernia diameter was $1.8 \pm 0.8 \mathrm{~cm}$. Mean operative time was $36.2 \pm 15.6 \mathrm{~min}-$ utes, with a mean mesh positioning time of $8.1 \pm 3.4$ minutes. Surgeons reported satisfaction with mesh ease of use in $95 \%$ of surgeries. The cumulative hernia recurrence rate at 1 year was $2.8 \%$ (3/106). Numeric Rating Scale (NRS) pain scores showed improvement from $2.1 \pm 2.0$ at preoperative baseline to $0.5 \pm 0.7$ at 1 month postoperative $(P<0.001)$, and this low pain level was maintained at 12 months postsurgery $(P<0.001)$. The mean global Carolina's Comfort Scale $^{\circledR}$ (CCS) score improved postoperatively from $3.8 \pm 6.2$ at 1 month to $1.6 \pm 3.5$ at 6 months $(P<0.001)$. One patient was unsatisfied with the procedure.

Conclusion: This 1-year interim analysis using PCO-VP for primary umbilical and epigastric defects shows promising results in terms of mesh ease of use, postoperative pain, and patient satisfaction. Recurrence rate is low, but, as laparoscopic evaluation shows a need for patch repositioning in some cases, an accurate surgical technique remains of utmost importance.

Keywords: intraperitoneal mesh, epigastric hernia, umbilical hernia, pain

\section{Plain language summary}

A hernia is the protrusion of bowel or fat through a weakness in the abdominal wall. Small ventral hernias can occur at the navel or upper part of the abdomen. These small hernias are treated by surgical repair and implantation of a mesh textile for abdominal wall support; however, placement and fixation of the mesh implant can be technically challenging. The device used in this study was designed to assist in these challenges; it contains removable handles for proper mesh positioning, four mesh flaps for suture fixation, and an absorbable expander to facilitate mesh deployment. This study followed up $>100$ hernia repair patients for 1 year to assess patient complications and hernia recurrence after repair with the device. Thus far, patients reported experiencing low pain and complication rates within 1 year after their repair, and three patients 
had a hernia recurrence. These results suggest that the device is effective for most patients within the first year of implantation.

\section{Introduction}

Primary umbilical and epigastric hernias exhibit recurrence rates of up to $16 \%$ following suture repair. ${ }^{1}$ Clinical studies have demonstrated the advantage of mesh hernioplasty over suture repair, even for small midline hernias of $1-3 \mathrm{~cm} .^{2-4}$ Retrorectus and preperitoneal dissection for these small hernias can be technically difficult. Self-expanding mesh devices can be introduced through the defect into the peritoneal cavity through an incision at the level of the hernia. After self-deployment of the device, traction on the fixation points or straps should achieve appropriate and flat placement posterior to the hernia defect. These devices have been embraced by many surgeons as a quick and elegant technique.

Two popular polypropylene (PP) devices have different tissue separating barriers. One has an ePTFE patch sewn to $\mathrm{PP}$ as an antiadhesive barrier, and the other is a nine-layer composite mesh with oxidized cellulose as an antiadhesive barrier. However, clinical studies of both devices have revealed cases of inadequate deployment, serious complications, and higher recurrence rates compared to traditional retromuscular mesh placement..$^{5-7}$ To solve the problems of both inadequate deployment and flat alignment to the abdominal wall, a device containing a dual-facing three-dimensional monofilament polyester mesh was introduced. The device includes four flaps for fixation, instead of the two fixation points on other available mesh devices.

This PANACEA study is a 24-month international noncomparative prospective study of patients undergoing open ventral hernia repair with intraperitoneal positioning of Parietex $^{\text {TM }}$ Composite Ventral Patch (PCO-VP). The 1-year results of all 126 enrolled patients are presented in this article.

\section{Patients and methods}

This study was conducted in accordance with the Declaration of Helsinki (2008), the protocol, ICH-GCP guidelines, and ISO 14155-2011. Approval of the study protocol and amendments, proposed informed consent forms, written subject information, and other study-related documents were obtained from institutional review boards (IRBs) and independent ethics councils (ECs) for each investigational site. These ECs and IRBs were Central Ethic Committee: Ethisch Comité UZ Gent (site 1), VZW (nonprofit organization) AZ Maria Middelares Hospital Ethisch Comité (site 2), WZV den Olm (nonprofit organization) Imelda Hospital: Bonheiden Medisch Ethisch Comité (site 3), Region Hovedstafden Koncern Organization og Personale, De Viden
Skasetiske Komiteer 3400 Hillerod (site 4), METC Medical Ethics Review Committee: CCMO Central Committee on Research Involving Human Subjects (site 5), EPN Regional Ethical Review Board in Linköping: Department for Medical Research Assessment (site 6), PTV Foundation for Tor Vergata University Hospital, Rome: Independent Ethic Committee (site 7), MO IRB (formerly WIRB and Capital Region) (site 8), Western Institutional Review Board (sites 9, 10, and 12), and Monmouth IRB (site 11). Written informed consent was obtained from subjects before any study-specific activity was performed and at the time of enrollment in the study. The study was registered publically at clinicaltrials. gov (NCT01848184).

\section{Trial design and objective}

In this prospective international multicenter noncomparative cohort study, patients underwent small primary ventral hernia repair with intraperitoneal PCO-VP positioning. The objectives were to assess hernia recurrence at 24 months follow-up, safety, and feasibility.

\section{Test device}

PCO-VP (Medtronic, Trevoux, France) is a dual-facing mesh composed of a nonabsorbable three-dimensional monofilament polyester textile for abdominal wall reinforcement. It has a bioabsorbable hydrophilic collagen film to minimize visceral attachment, absorbable expanders to facilitate proper placement, and a fixation system with four flaps and two removable handles. PCO-VP is indicated for repair of primary ventral defects and is available with an $8.6,6.6$, or $4.6 \mathrm{~cm}$ diameter.

\section{Participants}

Consented patients aged $\geq 18$ years scheduled for primary ventral hernia repair via open approach were assessed for eligibility via a screening/baseline visit within 6 weeks of their procedure. Patients who required emergency surgery, were participating in other trials, were pregnant, had a history of hernia at the same location of repair, had a body mass index $(\mathrm{BMI})>35 \mathrm{~kg} / \mathrm{m}^{2}$, had an American Society of Anesthesiologist score $\geq 4$, or were unable to comply with follow-up visits were excluded.

\section{Operative technique}

General anesthesia was administered, at which time patients received one dose of intravenous antibiotics according to hospital practice. After opening the hernia sac, the fascial defect was measured to ensure sufficient mesh overlap. For very small defects, the surgeon was allowed to increase the fascial 
gap by $\leq 0.5 \mathrm{~cm}$ to facilitate mesh introduction. For hernias $<1 \mathrm{~cm}$ in diameter, any size of PCO-VP was recommended. For hernias $1-3 \mathrm{~cm}$ in diameter, 6.6 and $8.6 \mathrm{~cm}$ PCO-VP were recommended, and for hernias $>3 \mathrm{~cm}$ in diameter, $8.6 \mathrm{~cm}$ PCO-VP was recommended. Patients with fascial defect diameter $>4 \mathrm{~cm}$ were withdrawn from the study. Devices were deployed in the intraperitoneal position according to the instructions for use. Four double-armed nonresorbable or slowly resorbable stitches were applied for fixation. Following skin closure, the surrounding muscle tissue, subcutaneous tissue, and dermis were infiltrated with local anesthetic. Final mesh placement was examined by laparoscopy with a $5 \mathrm{~mm}$ trocar in 10 patients at the Ghent University Hospital site. Surgical repositioning to obtain satisfactory alignment of the mesh was recorded.

Clinical assessments were performed at discharge, 1-, 6-, 12-, and 24-month follow-ups. Follow-up at 10 days was done by telephone.

\section{Outcomes measures}

The primary end point, recurrence at 24 months, was not assessed in this interim evaluation. The secondary end points were recurrence at 1 month ( -1 and +2 weeks), 6 months ( \pm 4 weeks), and 12 months ( -1 and +4 weeks) as diagnosed during a physical examination and confirmed by ultrasonography. Additional outcomes included 1) postoperative pain measured by the Numeric Rating Scale (NRS) 0-10 and compared to baseline values; 2) postoperative patient comfort measured by the Carolina's Comfort Scale ${ }^{\circledR}$ (CCS) (range: 0-115) calculated by the sum of sensation, pain, and movement scores; 3) accurate mesh alignment by laparoscopic exam in $n=10$ patients; and 4 ) adverse events (AEs).

\section{Statistics}

End points were represented by descriptive measures, and the sample size was determined by material, calendar, and site-recruitment constraints, and precision of measures and estimations desired. The recurrence rate for primary ventral hernia repair using synthetic mesh is $\sim 4 \%$ (range: 0\%-6\%). ${ }^{6,8-11}$ Assuming a 95\% confidence interval and $4 \%$ recurrence ( $\pm 5 \%$ precision), $\mathrm{N}=60$ patients were needed for the evaluable population. Anticipating a $20 \%$ lost to follow-up rate at both 12 and 24 months, a minimum of 100 patients were required as the evaluable population.

Data were summarized by counts, mean values, standard deviations, medians, minimum, and maximum (for continuous variables) or frequencies and percentages (for categorical variables). Recurrence rates and complications were analyzed on global follow-up and using time to event (Kaplan-Meier) analysis. Mean comparisons between subgroups were run using Student's $t$-test or nonparametric Mann-Whitney $U$-test. Proportion comparisons between subgroups were performed using chi-square or Fisher's exact test. All comparisons and tests were performed using two-sided tests with $\alpha$-level of 5\%. Analyses were performed using SAS ${ }^{\circledR}$ version 9.2 or higher (SAS Inc., Cary, NC, USA).

\section{Results \\ Demographics and procedural characteristics}

A total of 126 patients were enrolled between May 3, 2013, and July 10, 2014, at 12 centers in Europe and the US. The majority $(87.3 \%)$ of patients was treated for an umbilical hernia, while $12.7 \%$ were treated for epigastric hernia (Table 1). Mean BMI was $27.6 \pm 4.4 \mathrm{~kg} / \mathrm{m}^{2}$, with $34.1 \%$

Table I Patient demographics, comorbidities, and operative details, $\mathrm{N}=126$

\begin{tabular}{|c|c|}
\hline Age (years) & $51.2 \pm 12.2$ \\
\hline \multicolumn{2}{|l|}{ Gender } \\
\hline Female & $39(31.0)$ \\
\hline Male & $87(69.0)$ \\
\hline $\mathrm{BMI}, \mathrm{kg} / \mathrm{m}^{2}$ & $27.6 \pm 4.4$ \\
\hline$<30$ & $83(65.9)$ \\
\hline$\geq 30$ & $43(34.1)$ \\
\hline \multicolumn{2}{|l|}{ Hernia type } \\
\hline Umbilical & $110(87.3)$ \\
\hline Epigastric & $16(12.7)$ \\
\hline Subjects with at least one risk factor & $90(71.4)$ \\
\hline Smoker (current or prior) & $54(42.9)$ \\
\hline Obesity $\left(\mathrm{BMI} \geq 30 \mathrm{~kg} / \mathrm{m}^{2}\right)$ & $43(34.1)$ \\
\hline Diabetes type II & $10(7.9)$ \\
\hline COPD & $9(7.1)$ \\
\hline Stable disease & $6(66.7)$ \\
\hline Chronic disease requiring analgesic consumption & $2(1.6)$ \\
\hline Chronic disease requiring corticoid consumption & $3(2.4)$ \\
\hline \multicolumn{2}{|l|}{ Other disease leading to increased abdominal pressure } \\
\hline Benign prostatic hypertrophy & $\mathrm{I}(0.8)$ \\
\hline Liver cirrhosis/ascites/liver transplantation & $2(1.6)$ \\
\hline Mean hernia diameter, $\mathrm{cm}$ & $1.8 \pm 0.8$ \\
\hline \multicolumn{2}{|l|}{ Overlap, cm } \\
\hline$<1$ & $\mathrm{I}(0.8)$ \\
\hline $1.0-1.9$ & $43(34.1)$ \\
\hline $2.0-2.9$ & $71(56.3)$ \\
\hline $3.0-4.0$ & II (8.7) \\
\hline Mean \pm SD & $2.2 \pm 0.6$ \\
\hline Operative time, $\min (n=124)$ & $36.2 \pm 15.6$ \\
\hline Mesh positioning time, $\min (n=124)$ & $8.1 \pm 3.4$ \\
\hline \multicolumn{2}{|l|}{ Hospital length of stay, days } \\
\hline Median & 0.0 \\
\hline Min-max & $0-2$ \\
\hline
\end{tabular}

Notes: Data are presented as $\mathrm{n}(\%)$, mean \pm standard deviation, min-max, or median. Overlap $=($ size of mesh - hernia diameter $) / 2$.

Abbreviations: BMI, body mass index; COPD, chronic obstructive pulmonary disease; SD, standard deviation; min, minimum; max, maximum. 
of patients having a BMI over $30 \mathrm{~kg} / \mathrm{m}^{2}$. Risk factors at baseline (Table 1) included smoking (42.9\%), type II diabetes $(7.9 \%)$, and chronic obstructive pulmonary disease (7.1\%). Mean operative time was $36.2 \pm 15.6$ minutes, with a mean mesh positioning time of $8.1 \pm 3.4$ minutes. Ninety-six (76.2\%) patients were discharged the same day as the procedure, $25(19.8 \%)$ patients were discharged after 1 day, and 5 (4.0\%) patients were discharged after 2 days.

\section{Patient disposition and recurrence}

One-hundred ten patients were assessed at 12 months (Figure 1). Of the 110 patients, 5 were assessed too early (according to the protocol for the 12-month visit window) and thus were not included in the calculation for recurrence; these five patients had no recurrence at their last visit. In total, three hernia recurrences occurred within 12 months (at 79, 197, and 288 days after surgery), with a 1-year cumulative recurrence rate of $2.8 \%(3 / 106$, including the patient with recurrence at 79 days who withdrew from the study). Reoperation was performed for the patient with the earliest recurrence.

Protocol recommendations for mesh sizes were followed for 107/126 (84.9\%) patients. All patients who experienced recurrence had received the recommended size patch according to the hernia diameter (Table 2). There was no significant difference between the patch overlap (mesh-hernia diameter)

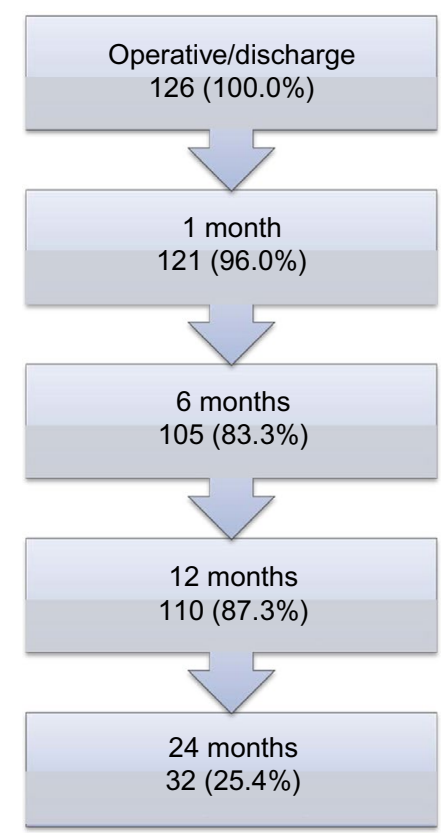

Figure I Patient follow-up visit completion.

Notes: The number of patients who successfully completed each visit is shown as $\mathrm{n}$ (\%). Twelve (9.5\%) patients withdrew early from the study. Early end-of-study reasons included: loss to follow-up $(n=4)$, adverse event $(n=1)$, withdrawal by subject $(n=6)$, and patient was unable to participate in follow-up visits $(n=1)$.
Table 2 Mesh size recommendations and recurrence

\begin{tabular}{lllll}
\hline \multirow{2}{*}{ Mesh size, cm } & \multicolumn{4}{l}{ Hernia diameter, cm } \\
\cline { 2 - 5 } & $<\mathrm{I}$ & $\mathbf{1 . 0 - 1 . 9}$ & $\mathbf{2 . 0 - 2 . 9}$ & $\mathbf{3 . 0 - 4 . 0}$ \\
\hline $4.6(\mathrm{~N}=39)$ & $\mathbf{8 ( 2 0 . 5 )}$ & $\mathbf{2 2 ( 5 6 . 4 ) ^ { \mathrm { a } }}$ & $8(20.5)$ & $\mathrm{I}(2.6)$ \\
$6.6(\mathrm{~N}=66)$ & $3(4.5)$ & $\mathbf{3 9}(59.1)$ & $\mathbf{1 8 ( 2 7 . 3 )}$ & $6(9.1)$ \\
$8.6(\mathrm{~N}=2 \mathrm{I})$ & $0(0.0)$ & $\mathrm{I}(4.8)$ & $10(47.6)^{\mathrm{a}}$ & $10(47.6)^{\mathrm{a}}$ \\
\hline
\end{tabular}

Notes: Data are presented as $\mathrm{n}(\%)$. Bold text indicates that the recommended mesh size for the corresponding defect size was used. ${ }^{a}$ The subsets that include a patient who exhibited hernia recurrence.

in patients with recurrence (mean: $2.6 \pm 0.8 \mathrm{~cm}$ ) versus those without recurrence (mean: $2.2 \pm 0.6 \mathrm{~cm})(P=0.336)$.

\section{Pain and quality of life}

Postoperative NRS scores are depicted in Figure 2. By day 10 postoperative, no patients were experiencing severe pain compared to $4(3.3 \%)$ and $5(4.1 \%)$ patients at baseline and discharge, respectively. At baseline and discharge, only 34/120 $(28.3 \%)$ and $17 / 123(13.8 \%)$ patients, respectively, were experiencing no pain, which improved to $77 / 123(62.6 \%)$ at 1 month and $93 / 111(83.8 \%)$ at 12 months. Compared to baseline, mean pain scores were reduced significantly $(P<$ $0.001)$ at 1,6 , and 12 months $(-1.6 \pm 2.0,-1.7 \pm 1.9$, and $-1.9 \pm 1.9$, respectively). The mean CCS score was $3.8 \pm$ 6.2 at 1 month postoperative, and it decreased significantly to $1.6 \pm 3.5$ and $1.4 \pm 4.3(P<0.001)$ at 6 and 12 months postsurgery, respectively. A vast majority of patients were completely satisfied with the procedure at all points of assessment (Figure 3).

\section{Mesh placement}

Surgeons reported a perioperative assessment of mesh manipulability and ease of use and were completely satisfied or satisfied with the ease of mesh handling in 120 (95.2\%) cases. In one $(0.8 \%)$ case, the surgeon reported complete dissatisfaction because the mesh had to be removed twice due to uncertain positioning of the device.

A laparoscopic examination was performed in the final 10 consecutively enrolled patients to assess mesh positioning following open repair. Two assessments were made: whether the mesh was in flat alignment, which was defined as the mesh lying in complete contact with the peritoneum with no spaces, and whether the mesh showed cupping, which was defined as any kink in the mesh (a so-called potato chip defect). Flat alignment was noted in the majority of cases (6/10 [60\%] patients). Interpositioning of fatty tissue prevented flat alignment in two patients (20\%), and cupping of the mesh at the side of the falciform ligament prevented flat alignment in two patients $(20 \%)$. In these four cases, the mesh position was 
A NRS pain by severity

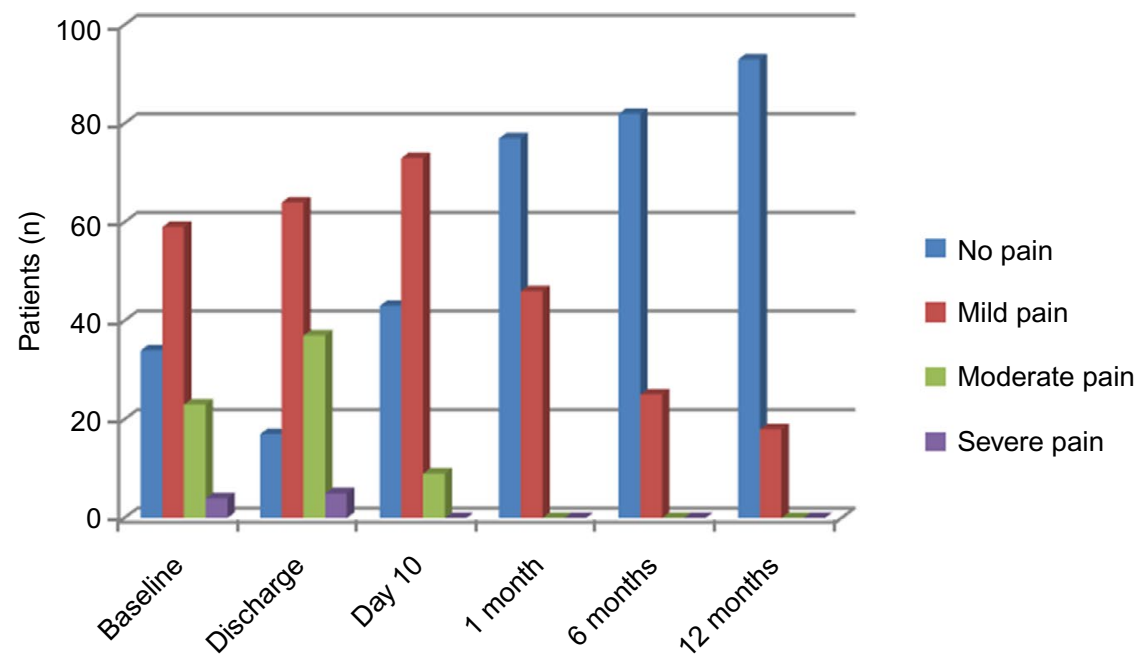

B

Mean NRS pain assessment

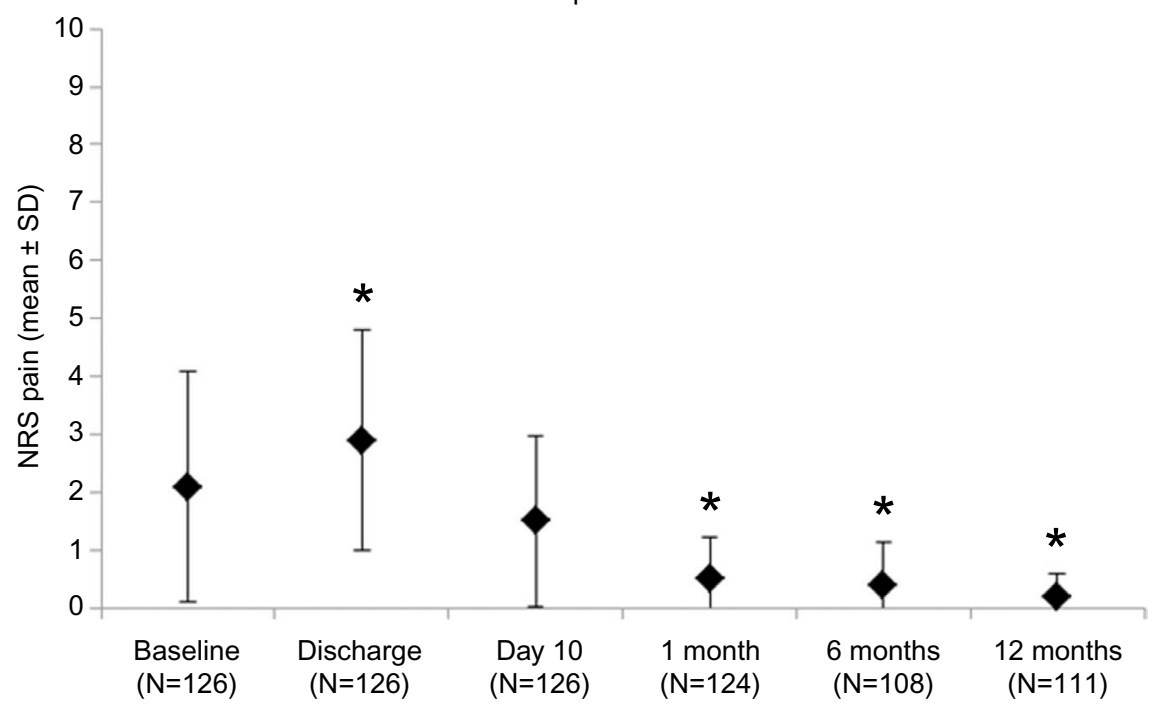

Figure 2 NRS pain assessment.

Notes: Pain levels were classified based on NRS scores: $0=$ no pain, $1-3=$ mild pain, $4-6=$ moderate pain, and 7-10 = severe pain. (A) Patient pain levels at baseline, discharge, day 10, I-, 6-, and I2-months postoperative. (B) Mean pain levels reported through 12 months. Standard deviation is shown. $* P<0.001$ relative to baseline. Abbreviation: NRS, Numeric Rating Scale.

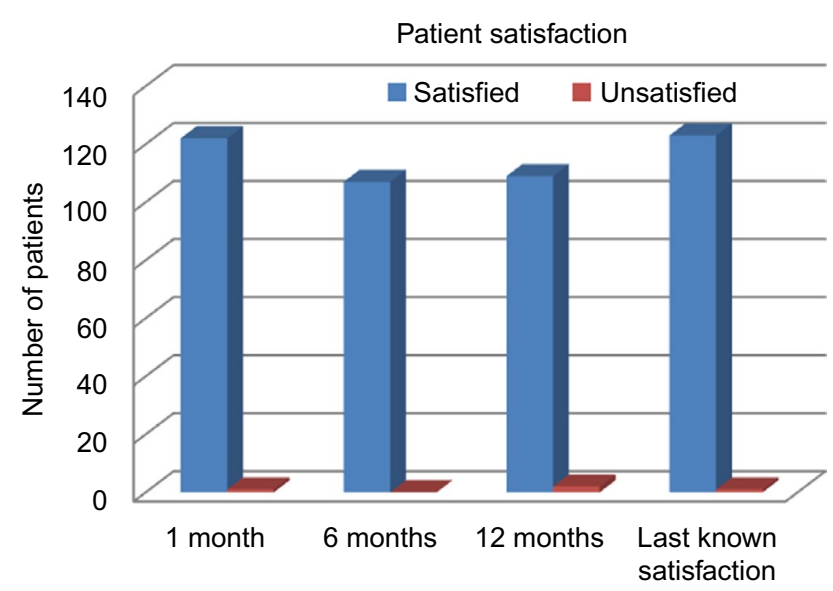

Figure 3 Patient satisfaction at I-, 6-, and I2-months postoperative and last known satisfaction for each patient. readjusted once each to achieve a completely flat alignment, without necessitating removal of the patch from the defect.

\section{Complications and AEs}

Fifty-nine AEs were experienced by 39 (31.0\%) patients, most (29) of whom had mild or moderate AE. Eighteen device-related $\mathrm{AE}$ occurred in 16 patients, including three serious $\mathrm{AE}$ in one patient each (procedural pain, pain due to recurrence, and recurrent umbilical hernia). Procedural pain was the most common (16 patients; $12.7 \%$ ) of all procedural- and device-related AE (Table 3). One (0.8\%) patient sustained a nonserious AE of a puncture of the small bowel during operation, two $(1.6 \%)$ patients developed incision site hematomas, and two (1.6\%) patients developed 
Table 3 Procedure- and device-related adverse events, $N=126$

\begin{tabular}{ll}
\hline Procedural pain & I6 (I2.7\%) \\
Wound infection & $5(4.0 \%)$ \\
Hernia recurrence & $3(2.4 \%)$ \\
Postoperative hematoma at the incision site & $2(1.6 \%)$ \\
Seroma & $2(1.6 \%)$ \\
Abdominal pain due to recurrence & $\mathrm{I}(0.8 \%)$ \\
Constipation & $\mathrm{I}(0.8 \%)$ \\
Fever & $\mathrm{I}(0.8 \%)$ \\
Hemoglobin down versus standard & $\mathrm{I}(0.8 \%)$ \\
Hypotension & $\mathrm{I}(0.8 \%)$ \\
Nausea & $\mathrm{I}(0.8 \%)$ \\
Occasional feeling of tightness at the operative site & $\mathrm{I}(0.8 \%)$ \\
Serous drainage at the incision site & $\mathrm{I}(0.8 \%)$ \\
Skin irritation & $\mathrm{I}(0.8 \%)$ \\
Swelling of left cheek & $\mathrm{I}(0.8 \%)$ \\
Puncture of small bowel & $\mathrm{I}(0.8 \%)$ \\
Vaginal bleeding & $\mathrm{I}(0.8 \%)$ \\
\hline
\end{tabular}

a seroma, all procedure related. Analysis showed no correlation between preoperative risk factors and seroma, wound infection, recurrence, or hematoma. Laparoscopic evaluation of a suspected recurrence at 15 months postsurgery revealed a preperitoneal lipoma; no recurrence was present. One surgeon reported a device malfunction, stating that the mesh did not open fully and had to be removed. The fascial incision was enlarged by $1.5 \mathrm{~cm}$, and a second mesh was deployed. In total, $31(27.2 \%)$ patients had their hernia gap enlarged as permitted by protocol to allow smooth introduction of the PCO-VP.

\section{Discussion}

Umbilical and epigastric hernias were historically repaired using primarily suture closure. Suture repair or the Mayo technique for umbilical hernia closure are both associated with a recurrence rate of $10 \%-30 \% .^{12,13}$ The use of a prosthetic mesh, even for small defects, significantly reduces hernia recurrence. ${ }^{2,3,14}$ This 1 -year interim analysis of the PANACEA trial shows a low 12-month recurrence rate of $2.8 \%(3 / 106)$. In addition, patients report minimal discomfort and high satisfaction following open repair with PCO-VP.

Historically, poor conformability to the parietal peritoneum has been a challenge, potentially leading to a cupping phenomenon or potato chip deformities. ${ }^{5,15}$ For some devices, the origin of these complications seems to be the excessive traction on the two straps that need to be pulled in order to draw the patch tightly against the parietal peritoneum. ${ }^{16}$ Extensive traction results in cupping and deformity of the patch that is unnoticed externally by the surgeon and only visible by laparoscopic evaluation after patch placement. ${ }^{5,17}$
Experimental and clinical studies of other devices have shown difficulties and recurrences likely due to extensive shrinkage of the different layers of materials in other patches. ${ }^{6,15,17}$

A recent study assessed the prosthetic design of PCO$\mathrm{VP},{ }^{18}$ which includes four mesh flaps, instead of only two straps, to aid in positioning and fixation. This design allows the patch to be deployed and stretched as much as possible by suturing the four flaps to the outer line of the covered fascial edges circumferentially. The authors concluded, after comparing Ventralex ${ }^{\mathrm{TM}}$ ST hernia patch (Bard Davol), Proceed ${ }^{\circledR}$ Ventral Patch (Ethicon), and PCO-VP, that both the intraperitoneal positioning and the incorporation of the $\mathrm{PCO}-$ VP mesh in the abdominal wall were improved compared to the other devices. ${ }^{18}$ In the case of PCO-VP, the antiadhesive collagen barrier seems less prone to intraperitoneal adhesions versus the oxidized regenerated cellulose barrier and has already proven its efficacy and safety in preclinical and clinical settings. ${ }^{19-21}$

Within our study, we observed misalignment of the mesh in 4 of 10 patients who underwent laparoscopic examination; however, the laparoscopic examination included only a small sample of our total study population, and thus the true rate of misalignment within our study may be lower or higher than the $40 \%$ observed.

This study is the first to extensively document clinical data for the new PCO-VP. Although not directly comparable due to differences in follow-up time and experience of surgeons, the observed $2.8 \%$ recurrence rate is less than that reported previously with use of the Ventralex ${ }^{\mathrm{TM}}$ hernia patch (up to $14.8 \%$ in 25 months $)^{5,9,16,22-25}$ or the Proceed ${ }^{\circledR}$ Ventral Patch (up to $12 \%$ in 16 months) ) $^{26-30}$ or by the regional cohort study from the Danish Hernia Database (up to $10 \%$ over 43 months) ${ }^{1}$ however, it is in line with the results from the meta-analysis by Nguyen et $\mathrm{al}^{31}$ showing a $2.7 \%$ recurrence rate after mesh repair of primary ventral hernias. These initial PANACEA study results are reported at 1 year, and a longer follow-up is necessary to assess whether this low recurrence rate with PCO-VP is maintained long term. Furthermore, as this study reports on many patient outcome parameters, all possible related complications have been documented herein. Historically, wound morbidity has been considered as one of the major disadvantages using mesh for repair of small umbilical and epigastric hernias. Hernia recurrence is lower using mesh but at the cost of a higher rate of wound morbidity and mesh infections compared to suture repair. ${ }^{31}$ Our study showed only $4.0 \%$ incidence of superficial wound infection, 
with no evidence of mesh infection. Since superficial wound infection only rarely leads to mesh infection, and since mesh repair of umbilical and epigastric hernias has been shown to have lower rates of recurrence than sutured repair, we conclude that mesh should be used for the repair of umbilical and epigastric hernias of any size, even very small ones of $<4 \mathrm{~cm}$. Furthermore, these 1-year results revealed no differences between epigastric and umbilical hernia in terms of dissection difficulty, mesh ease of use during the procedure, operative time, or incidence of AEs. The ratio of epigastric to umbilical hernia repair was, however, 16:110, and thus possible differences may not be exposed by our study.

By postoperative day $10,7.2 \%$ of patients were experiencing moderate pain, with the remainder experiencing mild or no pain. At 1 month, no patient reported moderate or severe pain, a rate maintained at 12 months. These results suggest a potentially better outcome than results with the Proceed ${ }^{\circledR}$ Ventral Patch, which showed 3.1\% (3/96) of patients still had moderate or severe pain at 12 months postsurgery; ${ }^{27}$ however, these differences may be due to different fixation methods or other study variables. In our study, this excellent patient outcome is also reflected in the quality-of-life global CCS score, which incorporates the sensation, pain, and movement scores. In contrast to results reported by others, only one of three patients experienced any pain associated with recurrence. Our laparoscopic intraperitoneal evaluations suggest that surgical technique using PCO-VP still remains crucial, particularly complete dissection of the falciform ligament cranially and the umbilical folds caudally to limit interference with the patch placement. This seems to be especially true for the larger patch of $8.8 \mathrm{~cm}$ diameter.

The study is limited by the absence of a control group, preventing conclusions about clinical performance of PCOVP compared to alternate devices or surgical mesh repair techniques. In addition, this interim publication does not report sufficient follow-up to draw conclusions about longterm safety and efficacy.

\section{Conclusion}

This closely monitored clinical trial has shown low recurrence rates thus far, with very low, and mainly mild morbidity 12 months postsurgery. The mesh device seems promising, with high postoperative QOL and excellent patient satisfaction.

\section{Acknowledgments}

Study sponsorship, funding, and data analysis were provided by Medtronic (Mansfield, MA, USA). The authors thank the study coordinators at each institution and the following individuals for assisting with the study: Tina Lee Broendum, RN, Claudi Mosconi, MD; Iris Kyle-Leinhase, PhD; Jeroen Ponten, MD; and Aude Vanlander, MD. Theresa K. Griffin, DC, and Emily L. Putiri, $\mathrm{PhD}$ (Medtronic), provided medical writing assistance under the authors' direction and based on content and conclusions developed by the authors; Patrice Becker and Djamila Bennat (Medtronic) provided editorial assistance based on the content and conclusions developed by the authors; and Sylvain Anselme (Medtronic) provided statistical analysis. All authors (or their institutions) received research support (financial and nonfinancial) from Medtronic to conduct this study.

\section{Author contributions}

All authors made substantial contributions to study conception and design (FB, FM, MGM, and LNJ), recruitment and data acquisition (FB, CD, FM, SH, MGM, SN, EK, TT, MRS, $\mathrm{KL}, \mathrm{VV}$, and LNJ), and/or data analysis and interpretation (FB). All authors participated in drafting the manuscript (FB, FM, and LNJ) and/or revising it critically for important intellectual content (FB, CD, FM, SH, MGM, SN, EK, TT, MRS, KL, VV, and LNJ). All authors gave final approval of the final manuscript version submitted to be published (FB, CD, FM, SH, MGM, SN, EK, TT, MRS, KL, VV, and LNJ).

\section{Disclosure}

The authors report no conflicts of interest in this work.

\section{References}

1. Christoffersen MW, Helgstrand F, Rosenberg J, Kehlet H, Strandfelt $\mathrm{P}$, Bisgaard T. Long-term recurrence and chronic pain after repair for small umbilical or epigastric hernias: a regional cohort study. $\mathrm{Am} \mathrm{J}$ Surg. 2015;209(4):725-732.

2. Arroyo A, Garcia P, Perez F, Andreu J, Candela F, Calpena R. Randomized clinical trial comparing suture and mesh repair of umbilical hernia in adults. Br J Surg. 2001;88(10):1321-1323.

3. Aslani N, Brown CJ. Does mesh offer an advantage over tissue in the open repair of umbilical hernias? A systematic review and meta-analysis. Hernia. 2010;14(5):455-462.

4. Sanjay P, Reid TD, Davies EL, Arumugam PJ, Woodward A. Retrospective comparison of mesh and sutured repair for adult umbilical hernias. Hernia. 2005;9(3):248-251.

5. Berrevoet F, Van den Bossche B, de Baerdemaeker L, de Hemptinne B Laparoscopic evaluation shows deficiencies in memory ring deployment during small ventral hernia repair. World J Surg. 2010;34(7):1710-1715.

6. Moreno-Egea A, Carrillo-Alcaraz A, Soria-Aledo V. Randomized clinical trial of laparoscopic hernia repair comparing titanium-coated lightweight mesh and medium-weight composite mesh. Surg Endosc. 2013;27(1):231-239.

7. Tinelli A, Malvasi A, Manca C, Alemanno G, Bettocchi S, Benhidjeb T. Post-laparoscopic mesh in post-menopausal umbilical hernia repair: a case series. Minim Invasive Ther Allied Technol. 2011;20(5):290-295.

8. Ammaturo C, Bassi UA, Bassi G. Outcomes of the open mesh repair of large incisional hernias using an intraperitoneal composite mesh: our experience with 100 cases. Updates Surg. 2010;62(1):55-61. 
9. Berrevoet F, D’Hont F, Rogiers X, Troisi R, de Hemptinne B. Open intraperitoneal versus retromuscular mesh repair for umbilical hernias less than $3 \mathrm{~cm}$ diameter. Am J Surg. 2011;201(1):85-90.

10. Briennon X, Lermite E, Meunier K, Desbois E, Hamy A, Arnaud JP. Surgical treatment of large incisional hernias by intraperitoneal insertion of Parietex(R) composite mesh with an associated aponeurotic graft (280 cases). J Visc Surg. 2011;148(1):54-58.

11. Nardi MJ, Millo P, Brachet Contul R, et al. Laparoscopic incisional and ventral hernia repair (LIVHR) with PARIETEX composite mesh. Minim Invasive Ther Allied Technol. 2012;21(3):173-180.

12. Halm JA, Heisterkamp J, Veen HF, Weidema WF. Long-term follow-up after umbilical hernia repair: are there risk factors for recurrence after simple and mesh repair. Hernia. 2005;9(4):334-337.

13. Velasco M, Garcia-Ureña MA, Hidalgo M, Vega V, Carnero FJ. Current concepts on adult umbilical hernia. Hernia. 1999;3(4):233-239.

14. Christoffersen MW, Helgstrand F, Rosenberg J, Kehlet H, Bisgaard T. Lower reoperation rate for recurrence after mesh versus sutured elective repair in small umbilical and epigastric hernias. A nationwide register study. World J Surg. 2013;37(11):2548-2552.

15. Reynvoet E, Chiers K, Van Overbeke I, Troisi R, Berrevoet F. Intraperitoneal mesh devices for small midline hernias: mesh behavior in a porcine model. Hernia. 2015;19(6):955-963.

16. Martin DF, Williams RF, Mulrooney T, Voeller GR. Ventralex mesh in umbilical/epigastric hernia repairs: clinical outcomes and complications. Hernia. 2008;12(4):379-383.

17. Garcia-Moreno F, Sotomayor S, Perez-Lopez P, et al. Intraperitoneal behaviour of a new composite mesh (Parietex Composite Ventral Patch) designed for umbilical or epigastric hernia repair. Surg Endosc. 2014;28(12):3479-3488.

18. Garcia-Moreno F, Perez-Lopez P, Sotomayor S, et al. Comparing the host tissue response and peritoneal behavior of composite meshes used for ventral hernia repair. J Surg Res. 2015;193(1):470-482.

19. Aube C, Pessaux P, Tuech JJ, et al. Detection of peritoneal adhesions using ultrasound examination for the evaluation of an innovative intraperitoneal mesh. Surg Endosc. 2004;18(1):131-135.

20. Balique JG, Benchetrit S, Bouillot JL, et al. Intraperitoneal treatment of incisional and umbilical hernias using an innovative composite mesh: four-year results of a prospective multicenter clinical trial. Hernia. 2005;9(1):68-74.
21. Deeken CR, Faucher KM, Matthews BD. A review of the composition, characteristics, and effectiveness of barrier mesh prostheses utilized for laparoscopic ventral hernia repair. Surg Endosc. 2012;26(2):566-575.

22. Hadi HI, Maw A, Sarmah S, Kumar P. Intraperitoneal tensionfree repair of small midline ventral abdominal wall hernias with a Ventralex hernia patch: initial experience in 51 patients. Hernia. 2006;10(5):409-413.

23. Iversen E, Lykke A, Hensler M, Jorgensen LN. Abdominal wall hernia repair with a composite ePTFE/polypropylene mesh: clinical outcome and quality of life in 152 patients. Hernia. 2010;14(6): $555-560$.

24. Tollens T, Den Hondt M, Devroe K, et al. Retrospective analysis of umbilical, epigastric, and small incisional hernia repair using the Ventralex hernia patch. Hernia. 2011;15(5):531-540.

25. Vychnevskaia K, Mucci-Hennekinne S, Casa C, et al. Intraperitoneal mesh repair of small ventral abdominal wall hernias with a Ventralex hernia patch. Dig Surg. 2010;27(5):433-435.

26. Ambe P, Meyer A, Kohler L. Repair of small and medium size ventral hernias with a Proceed Ventral Patch: a single center retrospective analysis. Surg Today. 2013;43(4):381-385.

27. Bontinck J, Kyle-Leinhase I, Pletinckx P, Vergucht V, Beckers R, Muysoms F. Single centre observational study to evaluate the safety and efficacy of the Proceed Ventral Patch to repair small ventral hernias. Hernia. 2014;18(5):671-680.

28. Muysoms FE, Bontinck J, Pletinckx P. Complications of mesh devices for intraperitoneal umbilical hernia repair: a word of caution. Hernia. 2011;15(4):463-468.

29. Tollens T, Struyve D, Aelvoet C, Vanrijkel JP. Introducing the Proceed Ventral Patch as a new device in surgical management of umbilical and small ventral hernias: preliminary results. Surg Technol Int. 2010; 19:99-103.

30. Wassenberg D, Zarmpis N, Seip N, Ambe PC. Closure of small and medium size umbilical hernias with the Proceed Ventral Patch in obese patients: a single center experience. Springerplus. 2014;3:686.

31. Nguyen MT, Berger RL, Hicks SC, et al. Comparison of outcomes of synthetic mesh vs suture repair of elective primary ventral herniorrhaphy: a systematic review and meta-analysis. JAMA Surg. 2014;149(5): 415-421.
Medical Devices: Evidence and Research

\section{Publish your work in this journal}

Medical Devices: Evidence and Research is an international, peerreviewed, open access journal that focuses on the evidence, technology, research, and expert opinion supporting the use and application of medical devices in the diagnosis, monitoring, treatment and management of clinical conditions and physiological processes. The identification of novel

\section{Dovepress}

devices and optimal use of existing devices which will lead to improved clinical outcomes and more effective patient management and safety is a key feature. The manuscript management system is completely online and includes a quick and fair peer-review system. Visit http://www. dovepress.com/testimonials.php to read real quotes from authors. 